\title{
Qualitative Analysis of the Implementation of a Hospital Room Service in a Large Metropolitan Hospital: Foundations for Transformation
}

\section{Shelley A. Wilkinson ( $\nabla$ s.wilkinson@uq.edu.au )}

The University of Queensland

Jack J. Bell

The University of Queensland

Bianca Neaves

The Prince Charles Hospital

Sally J. McCray

Mater Research Institute - University of Queensland

Katrina Campbell

Metro North Hospital and Health Service

\section{Research Article}

Keywords: barriers, enablers, food service, implementation, nutrition, room service

Posted Date: August 26th, 2021

DOl: https://doi.org/10.21203/rs.3.rs-820700/v1

License: (c) (1) This work is licensed under a Creative Commons Attribution 4.0 International License. Read Full License 


\section{Abstract}

Background: Room Service is a hospital meal service model with demonstrated improved nutrition intake, reduced wastage and cost benefits in some settings compared with traditional models. However, uptake across public hospital settings appears low; the underlying reasons require exploration. In 2019, Room Service was introduced in a Queensland Hospital and Health Service site. The aim of this paper is to identify the barriers and enablers to implementing Room Service to provide recommendations for future implementation of this model.

Methods: This qualitative descriptive study utilised semi-structured interviews with staff involved in implementation of the Room Service meal delivery model at The Prince Charles Hospital (Queensland, Australia). A purposive sample of project members and key stakeholders were recruited. Interviews explored project experiences from commencement to completion, barriers and enablers to implementation, strategies to overcome challenges and recommendations for implementation at other sites. Interviews were coded to identify themes and sub-themes.

Results: Nine participants were interviewed. Key themes with associated sub-themes were (I) Foundations of transformation; (II) Navigating implementation; and (III) Embedding sustainable practices.

Conclusions: This study adds rich information to understand factors that support the implementation of a room service model in a large public hospital. Future implementation of room service should not only consider measuring quantifiable outcomes, but also the importance of qualitative descriptive studies surveying project members and key stakeholders to further explore experiences, barriers and enablers to implementation and develop strategies to overcome challenges to assist further sites implement this model.

\section{Background}

Hospital foodservice systems are complex clinical structures that are required to meet the medical and socio-cultural needs of diverse clinical populations. Room Service is a contemporary hospital meal service model whereby patients order meals on demand at a time that suits them, utilising a bedside phone through to a call centre or via an electronic ordering tool such as bedside entertainment system or own personal device.

Room Service has primarily been implemented in the United States in the past 18 years, (1-4) but more recently introduced in Australia from 2013. $(5,6)$ While the support for Room Service in improving patient nutritional intake, reducing food costs and waste and improving patient satisfaction is strong, (16) limited studies have been done to identify these trends in the Australian public sector. Increasingly, as accreditation standards focus on the comprehensive care of patients, including nutrition and hydration status, (7) hospitals and health services are considering contemporary, innovative food service models with a dual priority of reducing waste and cost-savings, as well as being patient-centred to meet the clinical nutritional requirements of a wide range of patients and improving the patient experience. 
As a result of increasing awareness of the benefits of room service, in 2019, this model was introduced in a large (635 bed) tertiary public hospital. Prior to the introduction of the cook fresh on demand Room Service model, the food service system was a bought-in frozen product, thaw-retherm model. The Room Service implementation project was planned and delivered according to the knowledge to action (KTA) framework - Action cycle (8). Two determinant frameworks(9) were applied when identifying key stakeholders for building working party membership (consolidated framework for implementation research (CFIR)(10)) and developing strategies in various workforces to influence behaviour change to the new system (theoretical domains framework (TDF) (11). Governance of the project involved the formation of Project board and Internal working party, coming together eleven months prior to implementation of the room service system and disbanded one month post-implementation. These groups worked in synergy with the existing Food and nutrition working party that and supported the activities of the two project groups.

The Project board had five members, including Executive Sponsor, directors of the service line, and the business manager of the operational service line (added later). The group met bi-monthly with the purpose of providing executive leadership and decision making on the room service project and establishment of a new model of care. The main objectives of the board's activities were to oversee the capital works and procurement of equipment, provide financial accountability, respond to escalated concerns from the Working Party (below), and approve new policies and procedures as required. The Internal working party had 22 members, including medical, nursing, allied health, consumer, information technology, administration, external consultant, food services, quality and safety, telecoms, human resources, media, and education. The group, chaired by the Team Leader of Food Services and the Project Lead, met monthly with its main objectives to provide leadership and direction in the implementation of room service from a broad range of impacting areas within the facility. The group was responsible for developing all operational Policies, Procedures and work guidelines, ensure compliance with food safety, provide an avenue for consumer co-design of processes and provide a forum for communicating and managing risks and issues. This group reported to the Project Board allowing an avenue for escalation of risks and concerns. The Food and nutrition working party is a historical group of multidisciplinary staff (medical, allied health, nursing, food service, consumer, quality and safety, Aboriginal and Torres strait Islander representative) who met bimonthly and discussed all aspects of food, nutrition, hydration and malnutrition for the hospital. Many of this group's members represented their respective areas on the Internal working party. This group meets bimonthly and monitored food service/ monitors room service activities.

A suite of evaluations were administered to investigate the impact of Room Service on key clinical outcomes including food waste and costs, patients' nutritional intake, patient satisfaction and meal quality standards.(12) In addition to the clearly documented benefits of the Room Service model, it is important to articulate and define barriers and enablers that may enhance or prevent the model's adoption to inform future dissemination and scalability. Careful exploration of the experiences and perceptions of those who have recently implemented a transformation to Room Service will yield valuable insights to inform and support both successful sustainability and spread into new sites. The 
aim of this paper was consequently to identify the barriers and enablers to implementing Room Service to provide recommendations for future implementation of this model.

\section{Methods}

This qualitative descriptive study utilised semi-structured interviews with staff involved in implementation of the Room Service meal delivery model in a Queensland (Australia) Metro North Hospital and Health Service site during 2019. This hospital is a 635-bed tertiary metropolitan hospital in Brisbane, Australia providing specialist heart and lung services, intensive care, transplant, orthopaedic, paediatric, cystic fibrosis, general surgical, general medical, and rehabilitation services. Prior to Room Service implementation, the facility provided meals using a thaw-reheat and assembly serve model from bulk frozen meals, centrally plated in the kitchen and delivered to outlying wards. This study was deemed exempt from ethics review by an institutional ethics committees Project ID 53967.

A convenience sample of participants was recruited from project members and key stakeholders, including food services management, medical, dietetic, nursing, speech pathology, external project consultants, education and training and media/communications officers. Eligible participants were invited to participate via email from the research team. Those who responded received a written explanation of the study and provided written consent.

Interviews were undertaken by two members of the research team (SW and KC) by telephone $(\mathrm{n}=8)$ and face to face $(n=1)$ based on participant preference. Both interviewers were external to the organisation and had no role in the implementation process. Four declined participation. Interviews explored project experiences from commencement to completion, barriers and enablers to implementation, strategies to overcome challenges and recommendations for implementation at other sites. Interviews were average 17.1 minutes (range: 15.4-24.0 minutes). With consent, interviews were recorded on a digital recorder and transcribed verbatim for thematic analysis. ${ }^{(13)}$ The researchers (SW and KC) independently coded two interviews, agreed upon a coding framework and coded half the remaining transcripts each, noting illustrative text segments. They classified, sorted and synthesized codes in all transcripts to derive a smaller number of themes and sub-themes which were shared with other investigators and agreed upon by discussion and consensus. Finally, themes were examined with reference to the study aims and implementation science framework to identify learnings for other sites.

\section{Results}

Nine of the 13 potential participants were recruited. The interviewees comprised of a group of both clinical and non-clinical staff who had been involved on the local working party to implement room service. They included representation from food service management, dietetics, speech pathology, medical, nursing managers, education and training staff and an external project consultant who was engaged to assist with implementation. Interviewees had varying levels of engagement in the project from subject matter expert consultation to operationalising the implementation. 
Three main themes were derived with reference to the study aims: (I) Foundations of transformation, (2) Navigating implementation, and (3) Embedding sustainable practices. The themes and sub-themes are outlined below and illustrated with representative quotes.

\section{Theme one: Foundations of transformation}

Foundations of transformation's first sub-theme was (I) environmental and cultural assessment. It was acknowledged that consultation needed to happen early and widely. It was recognised this was "not a food service project, but a hospital project" and as such led to the conclusion that a broad organisation/ facility wide environmental scan or cultural assessment exploring local barriers and enablers would be beneficial for future sites' room service roll-outs. Considerations of stakeholders extended from the proximal to more distal groups, including executive, food service, dietetics, medical, nursing, operations, speech pathology, pharmacy, administration, consumers, media/communications and information technology.

"she \{Dietitian Team Leader Food Services\} worked hard to get that buy in and building that case for change quite early so I think that's why we were able to get those results because of that planning process, engaging people. I've seen it implemented in some other hospitals and unless you have that buy in, there's a lot of nervousness around it and so there's that desire to sort of go back to some of those old ways" (Participant 9)

Engagement with relevant unions to also be considered early on, from the outset and well prior to implementation, as well as apprising the needs of the patients and the community.

"talking about that community expectation, you know a lot of people don't like to come into hospital, but if there is one less thing that they need to worry about, they don't need to worry about the food anymore, like I wonder if that improves even coming into hospital." (Participant 2)

Shortcomings in the process of consultation were noted to hinder a smoother implementation. It was felt that issues around menu planning could have potentially been overcome by ensuring the interdisciplinary steering committee had the correct stakeholder membership, with links to Executive and other decision makers for two-way information sharing. The interdisciplinary approach to the steering committee or working group also allowed the for the development of relationships and trust during the process.

The second sub-theme, (II) preparing for anxiety and tensions, acknowledged the expected trepidation that exists before any large change to a system,

"because it's a change in culture, and people aren't too flush with cultural change"(Participant 2)

"maybe I was expecting some of the points that I was apprehensive about, not really expecting, but I was a little bit unsure whether or not certain aspects of it would work. I work in a general surgical ward and diets are upgraded and down graded quite frequently, so ... I wasn't sure how that was going to work, 
because you know you might see the patient and then 2 seconds later they want to order a meal through Room Service but yet we haven't been able to change that in the system yet." (Participant 7)

However, this sub theme also encompassed strengths, recognised with stories of problem solving in allaying fears and taking everyone on the journey. For example, participants said breaking down and addressing problems systematically, and recognising the evidence behind Room Service, were enablers for its implementation and uptake.

"When I first heard about it before I knew much about it I thought 'oh my God they're never going to be able to do this because it sounds too complicated', but as I got to know it better I thought 'this is going to be fantastic' and 'that's a lot of work', and then I wasn't sure how successful it would be because you really need to get it to work right to make it successful, and then as we went along and broke all the problems down and fixed them.. and implemented it and then it worked and it was like 'that was fantastic' and then it was done."(Participant 8)

"when I saw what had been learnt from .. other sites that had done it and when I heard about what the evidence .. I could see the potential. So, for instance, the lack of waste and the .. improved nutrition for the patients (because they were actually getting what they wanted) and \{were\} more likely to eat it, and I could see the logic behind that so I didn't need much persuading once I saw the experience and the evidence.(Participant 5)

Participants discussed ideas on preparation required to help support culture change, including appropriate staff training and equipping change champions in each area,

"and we had given them a lot of training beforehand about difficult conversations and resistance you know, people who are resistant to change and how to deal with those people, which at the time I think the department thought we were absolute crazy people, like you know, 'why, why are you telling us all this?' which was actually vindicated because someone said that they'd used all of those skills in the first hour"(Participant 1)

The final sub-theme related to (III) structural and strategic considerations prior to implementation. These focussed on issues suggested to be beneficial in preparing the service for the complex change. First and foremost was engagement of the executive.

"because it certainly helped and we needed exec to be pushed, you know, up to (Hospital and Health Service) exec to get the money, so without that, you know, whole hearted support it wouldn't have been a success, so getting that super early I think is is key"(Participant 3)

This early engagement was thought to assist in securing essential support for resourcing the remodel of the kitchen facilities. Other early engagement that was noted to facilitate change was the engagement of an experienced external consultant, as well as a well-networked project officer for the project delivery. Participants also encouraged approaching the process with an "open mind and a positive attitude" in the lead up to the implementation, as well as ensuring the change process had adequate time given to it. 
The second key theme, Navigating implementation, contained the sub-themes of (iv) experiences and engagement and (v) the transition phase, and (vi) reorientation of the workforce. Despite the initial trepidation, the (iv) experiences and engagement were reported as overwhelmingly positive for patients, staff groups, personal satisfaction, and the system. Respondents reported feeling that patients were empowered and in control,

"because it's about patients feeling in control. Patients often, particularly frail older patients in hospital, feel very out of control because they're stuck in hospital, they can't do anything, they can't complete their own activities of daily living. It's very disempowering. So to be able to have a menu and make choices and have some control is really good for them." (Participant 4)

Interviewees reported that staff showed adaptability, ownership, and empowerment and felt proud, pleased and amazed at the results of the Room Service system and its outcomes.

"I think they feel quite proud serving this nice, new, fresh food and then I think they're energised by the fact that the feedback they're getting from the patients is just really positive."(Participant 2)

"for me the best thing is the compliments, the amount of compliments that we get is amazing."(Participant 6)

The positive feelings extended to staff's pride in the significantly reduced waste and satisfaction with a more evidence based, patient focused approach.

"after my ward rounds, I see empty trays and it's such a good thing to see empty trays" (Participant 4) "at the end of the day it is about patient centred care, we have ideas as clinicians and therapists about how we want to do things but at the end of the day it's the patient, the person's in the centre"(Participant 4)

Minor negative comments were noted, mentioned as 'hiccups' or 'glitches' that were 'ironed out' through adaptability, further problem solving and collaborative decision making.

The second sub-theme in Navigating implementation was ( $v$ ) the transition phase. The success of undertaking a mock trial prior to go-live was highlighted, with the benefits a simulation can bring,

"We actually did a mock trial where the nurses upstairs would order food and we would take it up, you know those sort of scenarios.. So we did that. All the cooks were involved in that, so well before going live they had a good understanding of how it would work. From reading dockets, to looking at allergens, to the cooking processes, all those sorts of things."(Participant 6)

However, it was suggested that a more formal 'transition phase' during the commencement of the implementation of Room Service should occur. This was felt to be required to allow an 'overlap' or stock 
transition phase in the kitchen and ward imprest stores. When asked about their experiences of the actual go-live, participants said the extra staffing that was put on to allow for adjustment to the new system (with extra 'bodies' as runners and problem solvers) was essential to the smooth transition. A specific example noted was having all dietitians present and available on the wards and in the call centre to assist and problem solve during this go-live phase. This heightened teamwork and pressure was considered to be 'team building' and needed a 'celebration' once it was implemented to acknowledge the work achieved.

Reflecting on the effectiveness of communication strategies the Hospital and Health Service utilised during the covid-19 pandemic, it was suggested that short videoconferences (town hall-style) with executive could be utilised for future rollouts as an effective information and update tool for all staff.

Interviewees also highlighted the process of managing (vi) reorientation of the workforce. In addition to the positive benefits that came from having a dedicated project officer, it was noted that there were more permanent staffing changes needed and early planning is required for this.

"The workforce changes and having enough time to recruit and train and work out the rosters, and, I think HR seemed to sort of drag their feet a bit on that. Um I mean we went live end of April, it was a year yesterday, and said to them we need early February, we need all the stuff signed off and they were really dragging the chain. I don't think they understood the complexity and the severity, even though they had been involved in the key stakeholder meetings quite early on, I don't think they really got the breadth of the scope of the impact from a workforce point of view. Itl is a significant impact"(Participant 9)

As well as adequate resources, particularly in regards to some of the key food service roles, consideration of timely back up support or backfill of people undertaking key roles was recommended. Suggestions included requiring higher level workforce to be able to reorient to higher duties as well as restructuring the workforce and shift coverage (for example, having two people making sandwiches at busier times). Earlier recruitment of staff for the new roles was also recommended to occur to allow plenty of time for training.

It was also noted that the implementation could also have been enhanced by continuing the Project Board meetings for a longer period post go-live,

"I suppose since the project board and the project officer didn't continue on as long as they should have ... probably in the end it probably just didn't happen as quickly and as smoothly as it could have I think. And there are you know there are little things that crop up in those three months you know, with equipment and menu and those sorts of things probably could have been ironed out a bit easier. Yeah, if it had been yeah, for that kind of month longer really and the board in particular I think as well"(Participant 1)

Participants noted the potential benefit of having dedicated time allocated post implementation to review the changes and support any further problem solving that was required before resuming business as usual, 
"And then I've tried to do my normal role, I couldn't go in there and fine tune a lot of stuff.. I couldn't sort of evaluate it, like where are we going right, going wrong. We didn't have any time to reflect, you know what I mean"(Participant 6)

\section{Theme three: Embedding sustainable practices}

The final theme was Embedding sustainable practices for future success. Types of ordering systems that allow families to assist patients to order were acknowledged as possibly helping to take the burden off staff,

"the patient's family can order for them, they can order remotely, plus the nurses can order by \{the alternate system\} so it just means that from a workload point of view there's less demand on the workflow"(Participant 9)

On a larger system scale, considering the potential rollout across several sites within a Hospital and Health Service, the possible benefits of utilising a central call centre with a core, shared menu was noted,

"to streamline the job, use similar menus, you know and in the end too you know, looking at we could have one call centre in the middle of \{the Hospital and Health Service\}, they don't have to be on-site here, and then we have one call centre that you know does that does every meal order, it's just a phone number."(Participant 6)

The importance of remembering to engage new staff at orientation was highlighted,

"engaging people on day one at their orientation program to let them know it's coming ... what it's going to look and feel like so they can expect it, so there is no surprises ... I'll get staff that that have been to an orientation program, come to me and say wow, you know. You advised us of this and you asked us to be open minded and and to embrace this and l've never seen anything as amazing as this in a hospital"(Participant 2)

Finally, comments addressed ward and unit areas in which room service was not considered suitable as part of this rollout (such as rehabilitation wards with shared dining rooms), and suggested undertaking patient-centred problem solving to facilitate its adoption into the future.

\section{Discussion}

This study explored the experiences and reflections of staff involved in the implementation of a room service model to inform the future adoption of the model in other hospitals. Broad support for the model was identified, despite initial trepidation, with anticipated benefits for patients, staff, and the hospital reputation and finances. The foundations required for successful change required an assessment of the environment and culture, and addressing and preempingt anxieties, particularly through stories of previous success. Potential for larger scale implementation across a number of facilities within a health service district was also considered. 
Our findings have highlighted foundations of transformation, navigating implementation, and embedding sustainable practices as key themes to consider when planning, implementing, and sustaining new, technology supported foodservice change within highly complex systems. The synergy of our findings with contemporary models and frameworks for implementation, sustainability, scale and spread of system change are apparent. However, these were not explicitly articulated in the delivery of the Room Service system.

Building the reason to change, and keeping the change going, through embedding cyclical actionreflection evaluation early in the implementation have been highlighted both in our results and elsewhere, as a foundation of transformation of systems to improve nutrition care. (14-18) Ensuring the technology was able to generate data to support accurate and efficient foodservice delivery as well as process and outcomes evaluation to demonstrate the ongoing value proposition, influence on Patient Reported nutrition Experience Measures (nPREMS), organisational and wider system advantages, was a key factor that was considered foundational to a successful room service implementation.(12)

Early stakeholder engagement is not only required as a foundation for transformation, but to inform cyclical, iterative co-designed change processes.(19-23) Our findings confirm that engagement is a key component of navigating implementation. This should consider individual adopters (eg. patients, health professional and operational staff), inner setting or organisational influencers (eg. wards and units, dietetics and foodservices departments, hospital executive), outer setting/wider system factors (funders, unions, policy makers, professional and accrediting bodies, politicians, and community expectations) and cultural factors. $(19,20)$ However, building a reason to change and engaging the relevant players are not adequate on their own to embed change into practice. Accounting for the broader climate, whether funding drivers, political agenda, peer pressure, workforce or pandemics, identifying the previously 'unknown' variables should be considered fundamental to improving nutrition care in hospitals. (14, 20) Similarly the actual product itself, in this case room service, needs to have adequate evidence and perceived worth and return on investment, relative advantage and feasibility, to move from 'a reason to change' to the process of embedding. $(20,24)$

Engaging, maintaining ongoing engagement, and re-engaging patients and a broad range of key stakeholders have been recognised as a key to sustainable practices both in our findings and elsewhere. (14) Our findings confirm works by others regarding successful system change, highlighting the need for iterative adaptation over time, continuing to measure and report outcomes, attention to adequate infrastructure and resourcing, strategies to strengthen organisational resilience, and being and staying visible to further support sustainability of the room service model. (14) Those targeting scale and spread should ensure the system is tailored to make it easier to spread, and leveraging off opportunities that arise. (14) Conversely, we have highlighted the need to consider when and where alternative models should be maintained or considered to support the overall successful uptake of room service as appropriate. (19) 
This study is not without limitations. Despite a range of partners and stakeholders interviewed, this was only a small proportion of those involved in the implementation process. However, in depth perspectives gained from those interviewed do still provide a rich insight and key learnings of the project. The small number of participants also precluded attributing roles to specific quotes, but this was not done to maintain anonymity. The findings may also reflect the specific local health service engaged for this implementation. However, the learnings do align with the wider implementation literature and are worth close consideration before room service is implemented at other sites. There is also the potential of social desirability influencing the participants' responses, although, with the interviewers not involved in the project and external to the health service there is a lower chance of this being the case. Further, while endusers who were not involved in the implementation, but who likely experienced some issues and had feedback on the early go-live (such as ward dietitians, ward nurses, food service staff, patients) were not interviewed as part of this study, they should be considered in future research. Additionally, the delivery and evaluation of future room service implementations would benefit from the guidance and rigour of the application of a formal implementation science methodology, such as the CFIR (10) or iPAHRIS (25).

\section{Conclusion}

Room service is a contemporary foodservice model, progressively being adopted in Australia due to increasingly documented positive patient and organisational outcomes associated with its implementation. This study adds rich information to understand factors that support the implementation of a room service model in a large public hospital. Findings suggest that early engagement of not only executive and key decision makers, but of all stakeholders impacted by the change, be facilitated to ensure smooth implementation. In addition to building confidence and capacity of staff involved in the transition through exposure to success stories from other sites, a formalised trial run could be a valuable addition to implementation, as well as consideration of a longer retention of the project team postimplementation to manage issues that arise. Future implementation of room service should not only consider measuring quantifiable outcomes, but also the importance of qualitative descriptive studies surveying project members and key stakeholders to further explore experiences, barriers and enablers to implementation, develop strategies to overcome challenges to assist further sites implement this model, as well as plan for ongoing sustainability.

\section{Abbreviations}

CFIR

Consolidated framework for implementation research

KTA

Knowledge to action

nPREMS Patient reported nutrition experience measures

TDF Theoretical domains framework 


\section{Declarations}

\section{Ethics approval and consent to participate}

Project ID 53967, reviewed by The Prince Charles Hospital Human Research Ethic Committee, was noted as compliant with the National Health and Medical Research Council guidance "Ethical Considerations in Quality Assurance and Evaluation Activities" 2014. It was therefore deemed exempt from full ethical review on the basis that it wasa quality assurance project (Exempted 17 May 2019).Staff interviewed as part of the project were informed participation was voluntary and all provided verbal informed consent to participate in recorded interviews.

\section{Consent for publication}

Not applicable.

\section{Availability of data and materials}

Please contact the corresponding author regarding data availability.

The datasets used and/or analysed during the current study are available from the corresponding author on reasonable request.

\section{Competing interests}

JB is a Medical Research Future Fund MRFF 2018 Next Generation Clinical Researchers Program - TRIP Fellow translating the SIMPLE program into healthcare settings including The Prince Charles Hospital. All other authors declare that they have no competing interests.

\section{Funding}

This research did not receive any specific funding. JB is supported by a Medical Research Future Fund 2018 Next Generation Clinical Researchers Program - TRIP Fellowship.

\section{Authors' contributions}

All authors have participated sufficiently in the article to take public responsibility for the content. A/Prof Shelley Wilkinson (corresponding author), Dr Jack Bell, Bianca Neaves, and A/Prof Sally McCray were responsible for study design, data interpretation and manuscript preparation. A/Profs Wilkinson and Campbell were also responsible for collecting and analysing data.

\section{Acknowledgements}

Thanks to all interview participants for generously sharing their time and insights. The authors would like to acknowledge Emily Twine for assisting with data collection. 


\section{References}

1. Wadden $\mathrm{K}$, Wolf B, Mayhew A. Traditional versus room service menu styles for paediatric patients. Can J Diet Pract Res. 2006;67(2):92-4.

2. Kuperberg K, Caruso A, Dello S, et al. How will a room service delivery system affect dietary intake, food costs, food waste and patient satisfaction in a paediatric hospital? A pilot study. $\mathrm{J}$ Foodservice. 2008;19(5):255-61.

3. Houlston A, Buttery E, Powell B. Cook to order: Meeting the nutritional needs of children with cancer in hospital Pediatr Nurs 2009; 21(4):25-7.

4. McLymont V, Cox S, Stell F. Improving patient meal satisfaction with room service meal delivery. J Nurs Care Qual. 2003;18(1):27-37.

5. McCray S, Maunder K, Krikowa R, et al. Room Service Improves Nutritional Intake and Increases Patient Satisfaction While Decreasing Food Waste and Cost. J Acad Nutr Diet. 2018;118(2):284-93.

6. McCray S, Maunder K, Barsha L, et al. Room service in a public hospital improves nutritionalintake and increases patient satisfaction while decreasing food waste and cost $\mathrm{J}$ Human Nutr and Diet. 2018;31(6):734-41.

7. Australian Commission on Safety and Quality in Health Care. Comprehensive care standard Australian Commission on Safety and Quality in Health Care; 2019 [cited 202121 January]. Available from: https://www.safetyandquality.gov.au/standards/nsqhs-standards/comprehensive-care-standard.

8. Straus S, Tetroe J, Graham I. Knowledge translation in health care. Moving from evidence to practice. Oxford: Wiley-Blackwell/BMJ Books; 2009.

9. Nilsen P. Making sense of implementation theories, models and frameworks. Implementation Science. 2015;10(1):53.

10. Damschroder $L$, Aron $D$, Keith $R$, et al. Fostering implementation of health services research findings into practice: a consolidated framework for advancing implementation science. Implementation science: IS. 2009;4:50.

11. Francis $\mathrm{JJ}, \mathrm{O}^{\prime}$ Connor $\mathrm{D}$, Curran $\mathrm{J}$. Theories of behaviour change synthesised into a set of theoretical groupings: introducing a thematic series on the theoretical domains framework. Implementation Science. 2012;7:35.

12. Neaves B, Bell J, Twine E, et al. Impact of room service on patient intake, plate and production waste, meal quality and patient satisfaction - a single site pre-post evaluation of implementation Nutrition \& Dietetics. under review. 
13. Creswell J, Poth C. Qualitative Inquiry and research design: Choosing among five approaches. 4th ed. Thousand Oaks California: Sage; 2018.

14. Laur C, Bell J, Valaitis R, et al. The Sustain and Spread Framework: strategies for sustaining and spreading nutrition care improvements in acute care based on thematic analysis from the More-2-Eat study.(Report). BMC Health Services Research. 2018;18(1).

15. Glasgow RE, Harden SM, Gaglio B, et al. RE-AIM Planning and Evaluation Framework: Adapting to New Science and Practice With a 20-Year Review. Front Public Health. 2019;7:64-.

16. Bell JJ, Rossi T, Bauer JD, et al. Developing and evaluating interventions that are applicable and relevant to inpatients and those who care for them; a multiphase, pragmatic action research approach. BMC Med Res Methodol. 2014;14:98.

17. Bell J, Young A, Hill J, et al. Rationale and developmental methodology for the SIMPLE approach: A Systematised, Interdisciplinary Malnutrition Pathway for impLementation and Evaluation in hospitals. Nutrition \& Dietetics. 2018;75(2):226-34.

18. Bell JJ, Young A, Hill J, et al. Systematised, Interdisciplinary Malnutrition Program for impLementation and Evaluation (SIMPLE) delivers improved hospital nutrition care processes and patient reported experiences - an implementation study Nutrition \& Dietetics.

2021,https://doi.org/10.1111/1747-0080.12663.

19. Greenhalgh T, Wherton J, Papoutsi C, et al. Beyond Adoption: A New Framework for Theorizing and Evaluating Nonadoption, Abandonment, and Challenges to the Scale-Up, Spread, and Sustainability of Health and Care Technologies. J Med Internet Res. 2017;19(11):e367.

20. Damschroder LJ, Aron DC, Keith RE, et al. Fostering implementation of health services research findings into practice: a consolidated framework for advancing implementation science. Implementation Science : IS. 2009;4:50-.

21. Laur C, Valaitis R, Bell J, et al. Changing nutrition care practices in hospital: a thematic analysis of hospital staff perspectives. BMC Health Serv Res. 2017;17(1):498.

22. Moore GF, Audrey S, Barker M, et al. Process evaluation of complex interventions: Medical Research Council guidance. BMJ : British Medical Journal. 2015;350:h1258.

23. Cathain A, Croot L, Duncan E, et al. Guidance on how to develop complex interventions to improve health and healthcare. BMJ Open. 2019;9(8):e029954.

24. Bowen DJ, Kreuter M, Spring B, et al. How we design feasibility studies. Am J Prev Med. 2009;36(5):452-7. 
25. Harvey G, Kitson A. PARIHS revisited: from heuristic to integrated framework for the successful implementation of knowledge into practice Implementation Sci 11, 33 (2015) https://doiorg/101186/s13012-016-0398-2. 2015;11:33. 